\title{
The biaxial mechanics of thermally denaturing skin - Part I: Experiments
}

\author{
William D. Meador ${ }^{\mathrm{a}}$, Gabriella P. Sugerman ${ }^{\mathrm{a}}$, Adrian Buganza Tepole ${ }^{\mathrm{b}, \mathrm{c}}$, \\ Manuel K. Rausch ${ }^{\mathrm{a}, \mathrm{d}, \mathrm{e}, *}$ \\ ${ }^{a}$ Department of Biomedical Engineering, The University of Texas at Austin, Austin, TX, \\ 78712, USA \\ ${ }^{b}$ School of Mechanical Engineering, Purdue University, West Lafayette, IN, 47907, USA \\ ${ }^{c}$ Weldon School of Biomedical Engineering, Purdue University, West Lafayette, IN, 47907, \\ USA \\ ${ }^{d}$ Department of Aerospace Engineering and Engineering Mechanics, The University of \\ Texas at Austin, Austin, TX, 78712, USA \\ ${ }^{e}$ Oden Institute for Computational Engineering and Sciences, The University of Texas at \\ Austin, Austin, TX, 78712, USA
}

\begin{abstract}
The mechanics of collageneous soft tissues, such as skin, are sensitive to heat. Thus, quantifying and modeling thermo-mechanical coupling of skin is critical to our understanding of skin's physiology, pathophysiology, as well as its treatment. However, key gaps persist in our knowledge about skin's coupled thermo-mechanics. Among them, we haven't quantified the role of skin's microstructural organization in its response to superphysiological loading. To fill this gap, we conducted a comprehensive set of experiments in which we combined biaxial mechanical testing with histology and two-photon imaging under liquid heat treatment. Among other observations, we found that unconstrained skin, when exposed to high temperatures, shrinks anisotropically with the principle direction of shrinkage being aligned with collagen's principle orientation. Additionally, we found that when skin is isometrically constrained, it produces significant forces during denaturing that are also anisotropic. Finally, we found that denaturation significantly alters the mechanical behavior of skin. For short exposure times, this alteration is reflected in a reduction of stiffness at high strains. At long exposure times, the tissue softened to a point where it became untestable. We supplemented our findings with confirmation of collagen denaturation in skin via loss of birefringence and second harmonic generation. Finally, we captured all time-, temperature-, and direction-dependent experimental findings in a hypothetical model. Thus, this work fills a fundamental gap in our current understanding of skin thermo-mechanics and will support future developments in thermal injury prevention, thermal injury management, and thermal therapeutics of skin.
\end{abstract}

\footnotetext{
* Corresponding author

Email address: manuel.rausch@utexas.edu (Manuel K. Rausch)
} 
Keywords: Planar Biaxial, Two Photon Microscopy, Histology, Shrinkage, Collagen, Birefringence, Mouse, Isometric, Isotonic

\section{Introduction}

All biological tissues, including collageneous soft tissues, are sensitive to heat. In fact, collagen, the predominant structural protein in collageneous soft tissues, denatures at temperatures significantly exceeding body temperature 1]. That

${ }_{5}$ is, at temperatures well above $37^{\circ} \mathrm{C}$ collagen's triple helix structure collapses as inter- and intra-molecular cross-links are broken, and the resulting unstructured collagen molecules dissociate [2]. Eventual loss of functional collagen transforms these tissues into gel-like, gelatinous materials. This transformation comes with a loss of load-bearing ability, i.e., stiffness and toughness [3]. Additionally, 10 soft tissues shrink significantly during denaturation [4. These processes are not instantaneous but follow exponential functions that demonstrate Arrheniustype temperature-dependence [5]. Previously, Chen et al. demonstrated this behavior for chordae tendineae, a highly collageneous cardiovascular soft tissue, and described time-temperature equivalence for this tissue [6]. In a later study,

${ }_{15}$ Chen et al. further demonstrated time-temperature-stress equivalence in similar collageneous tissues highlighting the multibiophysical character of collageneous soft tissue denaturation [7. One-dimensional, or uniaxial tests, where later extended by the same investigators to two-dimensional, or biaxial, studies of membranous materials. Those studies showed that time-, temperature-, and

20 load-dependence may be similarly observed as in above studies but also revealed that uniaxial data cannot be simply extrapolated to multi-axial data [8, 9].

Understanding thermally-induced denaturation of collageneous soft tissues is clearly important from a basic pathophysiological perspective. Additionally, understanding this biophysical process is critical from a therapeutic perspec25 tive. For example, Boronyak et al. and Price et al. proposed using thermal denaturation-induced shrinkage to treat myxomatous mitral valve regurgitation 10, 11, 12. Similarly, thermally-induced tissue shrinkage has been used to treat shoulder instability [13, 14] and to close femoral access wounds [15]. These are just a few of many applications where thermal denaturation of collageneous so tissues was applied therapeutically.

For skin specifically, studies of thermal denaturation are critical both from a pathophysiological perspective as well as from a therapeutic perspective. As skin's function is to protect us from environmental threats, such as heat, understanding and quantifying its response to superphysiological loading, i.e.,

35 thermally-induced denaturation, is vital [16, 17]. Similarly, there are skinspecific medical therapies that use thermal loading whose multibiophysical effects and limits should be known for safe use, such as laser-ablation [18. Finally, aesthetic trends such as branding make direct use of thermal denaturation, again, requiring knowledge of the underlying processes for safe application [19].

Because of skin's critical role in thermoregulation, there have been numerous studies investigating skin under physiological conditions with and without 
including effects on or due to mechanics [20, 21. On the other hand, studies of skin mechanics under superphysiological conditions are sparse. Notable exceptions include those by Zhou et al and $\mathrm{Zu}$ et al. for example [22, 23].

45 They demonstrated that thermally denaturing skin exhibits constitutive behavior that is temperature and load-rate dependent. However, there have been no reports of skin biaxial mechanics of thermally denaturing skin under both isotonic and isometric conditions that considered the tissue's anisotropy. The objective of our current work is to fill this gap. To this end, we conduct three

50 distinct experiments: i) isotonic, denaturation-induced shrinkage measurements at superphysiological temperatures, ii) isometric, denaturation-induced tension measurements at the same superphysiological temperatures, iii) and finally, biaxial test of skin's anistropic mechanical properties before and after heat treatment. We supplement and interpret these data by means of classic histology and two-photon microscopy-based structural investigations.

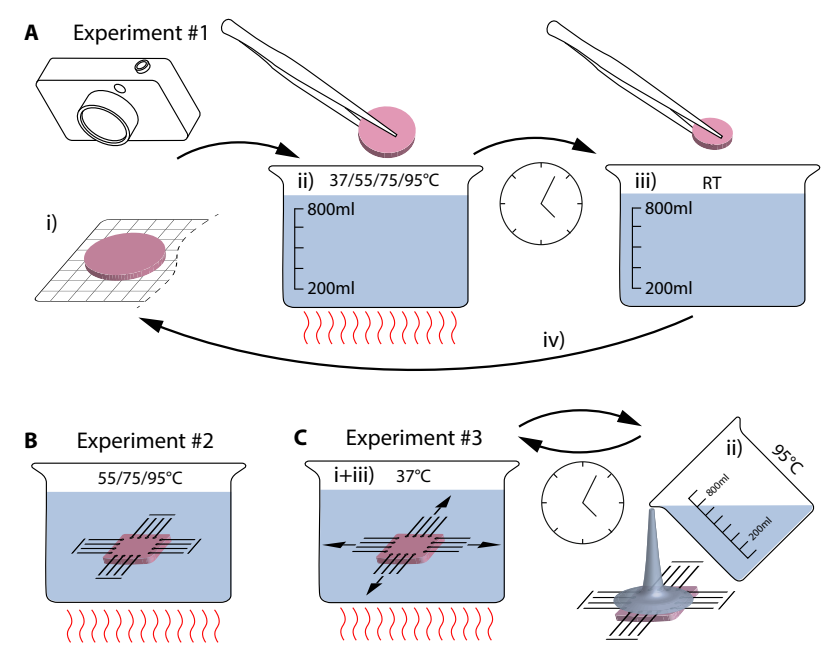

Figure 1: Experimental design of our study. A) Experiment \#1 describes our biaxial shrinkage study under traction-free or isotonic boundary conditions in which we i) took images of excised skin samples on a calibrated grid, before ii) submerging the samples in a $1 \mathrm{xPBS}$ bath heated to $37^{\circ} \mathrm{C}, 55^{\circ} \mathrm{C}, 75^{\circ} \mathrm{C}$, or $95^{\circ} \mathrm{C}$ for various exposure times. We halted the temperature treatment by iii) submerging samples in $37^{\circ} \mathrm{C}$ water before, iv) yet, again, taking images of the samples on a calibrated grid. B) Experiment \#2 describes our isometric biaxial experiment, in which we i) quantified the biaxial constitutive behavior of excised skin samples in a $37^{\circ} \mathrm{C}$ water bath, before ii) exchanging the bath with $55^{\circ} \mathrm{C}, 75^{\circ} \mathrm{C}$, or $95^{\circ} \mathrm{C}$ water for 5 minutes during which we measured the resulting forces while fixing the boundaries of the sample in its previously stress-free reference configuration. Subsequently, we iii) retested the biaxial constitutive behavior of the samples. C) Experiment \#3 describes our second biaxial experiment, in which we i) quantified the biaxial constitutive behavior of excised skin samples in a $37^{\circ} \mathrm{C}$ water bath, before ii) removing the water bath and treating the samples with a $90^{\circ} \mathrm{C}$ water shower for varying exposure times during which we measured the resulting forces while fixing the boundaries of the sample in its previously stress-free reference configuration. Subsequently, we iii) retested the biaxial constitutive behavior of the samples 


\section{Materials and Methods}

\section{Skin samples}

All animal experiments were conducted in accordance with NIH's Guide for Care and Use of Laboratory Animals and after approval from our local ${ }_{60}$ institutional animal care and use committee. All mice were humanely sacrificed via $\mathrm{CO}_{2}$ inhalation before we removed the hair from the dorsal skin regions with clippers and a chemical depilatory agent (Nair, Churd \& Dwight, Inc., Ewing, NJ, United States). For the isometric biaxial test samples, we applied an ink stamp with known dimensions $(6 \mathrm{~mm} \times 6 \mathrm{~mm})$ to four dorsal skin regions

65 before excising those regions as $10 \mathrm{~mm} \times 10 \mathrm{~mm}$ square samples. For the isotonic shrinkage and two-photon studies, we used biopsy punches to remove up to eight $6 \mathrm{~mm}$ dorsal skin samples.

\section{Isotonic shrinkage experiments}

First, we placed discoid skin samples of $6 \mathrm{~mm}$ diameter on a 1xPBS-wetted, calibrated grid and took photographs of the samples. Next, we submerged those samples in $37^{\circ} \mathrm{C}, 55^{\circ} \mathrm{C}, 75^{\circ} \mathrm{C}$, or $95^{\circ} \mathrm{C} 1 \times \mathrm{PBS}$ for varying durations. After removing the samples from the bath we immediately submerged the tissue in a $37^{\circ} \mathrm{C} 1 \mathrm{xPBS}$ bath, i.e., "quenched" the tissue. We then took photographs of those samples on a 1xPBS-wetted calibrated grid again. See Figure 11A for a depiction of the protocol.

\section{Isometric biaxial tension experiments}

We mounted the $10 \mathrm{~mm} \times 10 \mathrm{~mm}$ square samples onto our customized biaxial tensile tester (Cell Scale, Biotester, Waterloo, Canada) within 4 hours of excision. Once mounted, we established an approximately stress-free reference configuration by loading the tissue to an equibiaxial preload of $20 \mathrm{mN}$. Next, we conducted our standard biaxial protocol to equibiaxial force of $1000 \mathrm{mN}$ while the samples were submerged in $37^{\circ} \mathrm{C} 1 \mathrm{xPBS}$. We repeated this loading cycle 20 times until the material response stabilized. After recording the tissues baseline constitutive response, we conducted one of two protocols. During the first

85 set of experiments, we let the tissue recover from initial biaxial testing for 5 minutes in $37^{\circ} \mathrm{C} 1 \mathrm{xPBS}$. After this recovery period, we replaced the fluid bath with either $55^{\circ} \mathrm{C}, 75^{\circ} \mathrm{C}$, or $95^{\circ} \mathrm{C} 1 \mathrm{xPBS}$ and recorded the developing force under isometric tension, i.e., with fixed boundary conditions. We recorded force and temperature of all samples for the ensuing 5 minutes until the material response equilibriated. During the second, separate set of experiments, we also let the tissue recover for 5 minutes following the initial biaxial testing. After this period, we lowered the tissue bath so that the samples were suspended on our rake system and then gently poured $90^{\circ} \mathrm{C} 1 \mathrm{xPBS}$ for between 2-30 seconds onto the samples before re-submerging the samples in $37^{\circ} \mathrm{C} 1 \mathrm{xPBS}$ to abruptly stop

95 the heat treatment. We continuously recorded skin temperature with a thermal imaging camera (FLIR, A35/65, Wilsonville, OR, United States) and force while we poured $1 \mathrm{xPBS}$ onto our samples and during the following 5 minutes 
after quenching (see Supplementary Video 1 for a recording of this experiment). Following this second set of experiments, we repeated equibiaxial mechanical testing in 20 cycles to $1000 \mathrm{mN}$ of force. See Figure $1 \mathrm{~B}-\mathrm{C}$ for a depiction of the two protocols.

\section{Histology \& Two-photon microscopy}

We heat-treated $6 \mathrm{~mm}$ discoid skin samples in $37^{\circ} \mathrm{C}, 55^{\circ} \mathrm{C}, 75^{\circ} \mathrm{C}$, and $95^{\circ} \mathrm{C}$ $1 \mathrm{xPBS}$ for either 10 seconds or 5 minutes and subsequently fixed half of those samples in $10 \%$ formalin for 24 hours before shipping them in ethanol to a commercial histology service (Histoserv, Inc., Germantown, MD, United States). Histoserv stained our tissues with Masson's trichrome and Picrosirius red. Upon receipt of the stained tissue sections we imaged all samples on a $4 \mathrm{X}$ optical microscope (BX53, Olympus, Center Valley, PA, United States). The other half of the samples, we imaged under our two-photon microscope using our previously developed imaging protocol in which we excited the tissue under $900 \mathrm{~nm}$ and epi-collected the emission signal with a $460 \pm 25 \mathrm{~nm}$ filter to collect collagen's second harmonic generation signal at a resolution of 1024 by 1024 pixels (over a $500 \mu \mathrm{m} \times 500 \mu \mathrm{m}$ region of interest within the dermis of the skin 115 samples, see Figure 2. Here we followed our own and others' previous work [24, 25, 26].

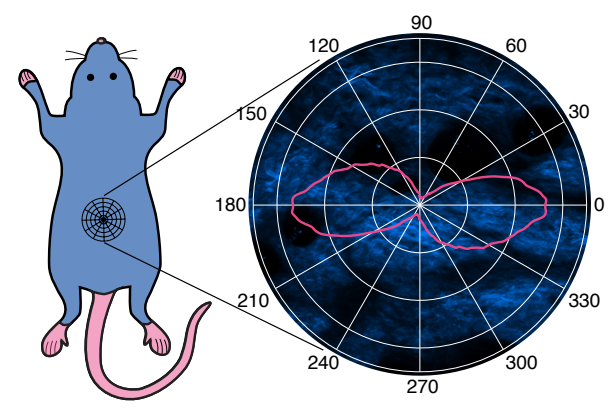

Figure 2: Representative second harmonic generation image of the collagen orientation in dorsal mouse skin. Based on an orientation distribution analysis in ImageJ, we also show the collagen orientation distribution function indicating a clear anisotropy with collagen's predominant orientation in lateral direction 27

\section{Data analysis}

First, we evaluated tissue shrinkage based on the photographs taken before and after heat treating in Experiment \#1. To this end, we used a custom Matlab program to digitize the perimeter of each sample and automatically quantified area shrinkage as the percentage area change following heat treatment. Additionally, we computed longitudinal and lateral strain as percentage width and height changes, respectively.

Second, we evaluated the biaxial constitutive behavior of skin before and after heat treatment in Experiments $\# 2$ and $\# 3$. To this end, we evaluated 
the force strain data during the downstroke of the last biaxial testing cycle. To compute Green-Lagrange strain, we conducted digital image correlation based on the stamp marks on the tissue samples. Next, we transformed force measurements into membrane tension based on the deformed sample width. For each 130 stress-strain curve, we evaluated "toe-stiffness", i.e., stiffness at small strains, and "calf-stiffness", i.e., stiffness at large strains by computing the respective tangent moduli.
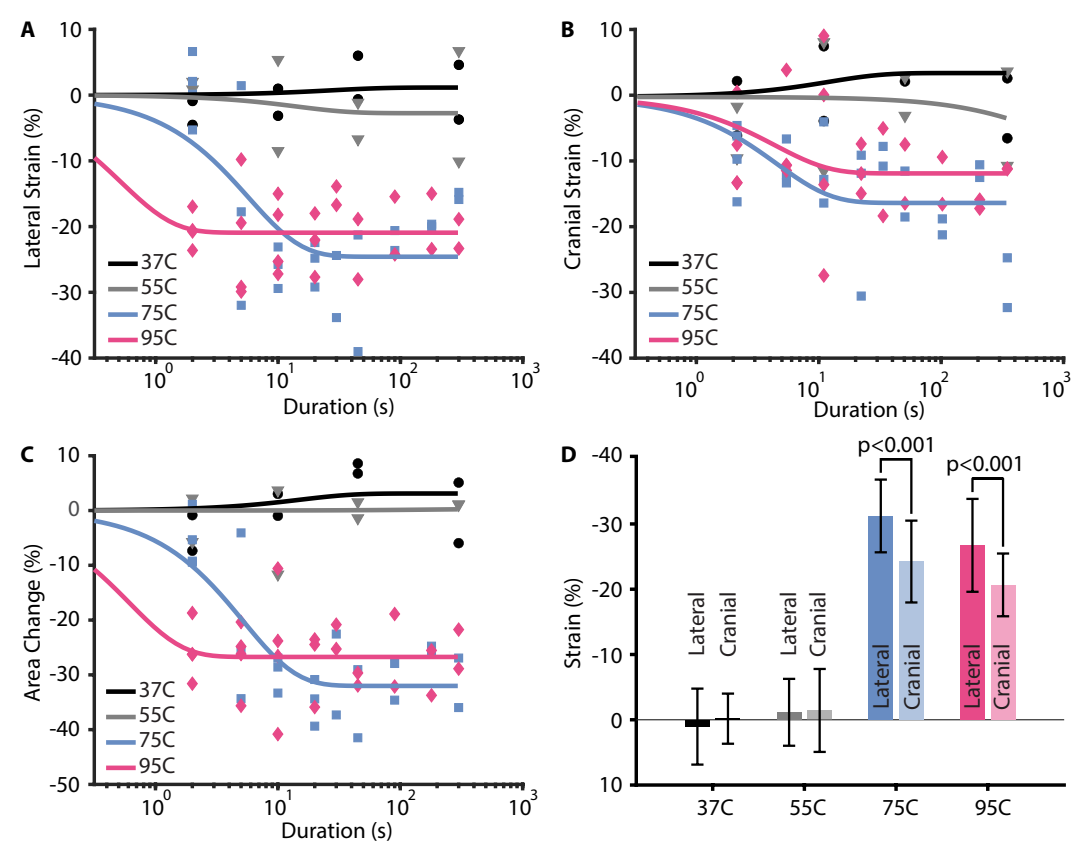

Figure 3: Heat treatment with $75^{\circ} \mathrm{C}$ and $95^{\circ} \mathrm{C} 1 \mathrm{xPBS}$ induces anisotropic shrinkage under isotonic conditions. A) Heat treatment with $37^{\circ} \mathrm{C}(\mathrm{n}=9)$ and $55^{\circ} \mathrm{C}(\mathrm{n}=9)$ did not induce significant lateral strain in discoid skins samples, while treatment with $75^{\circ} \mathrm{C}(\mathrm{n}=24)$ and $95^{\circ} \mathrm{C}(\mathrm{n}=26)$ did. Interestingly, skin shrunk faster at $95^{\circ} \mathrm{C}$ than at $75^{\circ} \mathrm{C}$, but reached similar equilibrium values after 5 minutes. B) Shrinkage in cranial direction mimicked the qualitative shrinkage behavior in lateral direction. However, quantitatively, skin shrunk more in lateral than in cranial direction (see D). C) Heat treatment-induced area shrinkage reflected the qualitative behavior in lateral and cranial directions. D) At equilibrium, $37^{\circ} \mathrm{C}$ and $55^{\circ} \mathrm{C}$ treatments did not result in shrinkage, while $75^{\circ} \mathrm{C}$ and $95^{\circ} \mathrm{C}$ treatments resulted in significant anisotropic shrinkage, most notably in the lateral direction

\section{Statistics}

All data are shown as mean \pm standard deviation and sample numbers are provided in each figure caption. We conducted all statistical analyses via linear mixed models as implemented in "R" (Version 4.0.3). Specifically, we used the afex library as we previously reported [28]. 

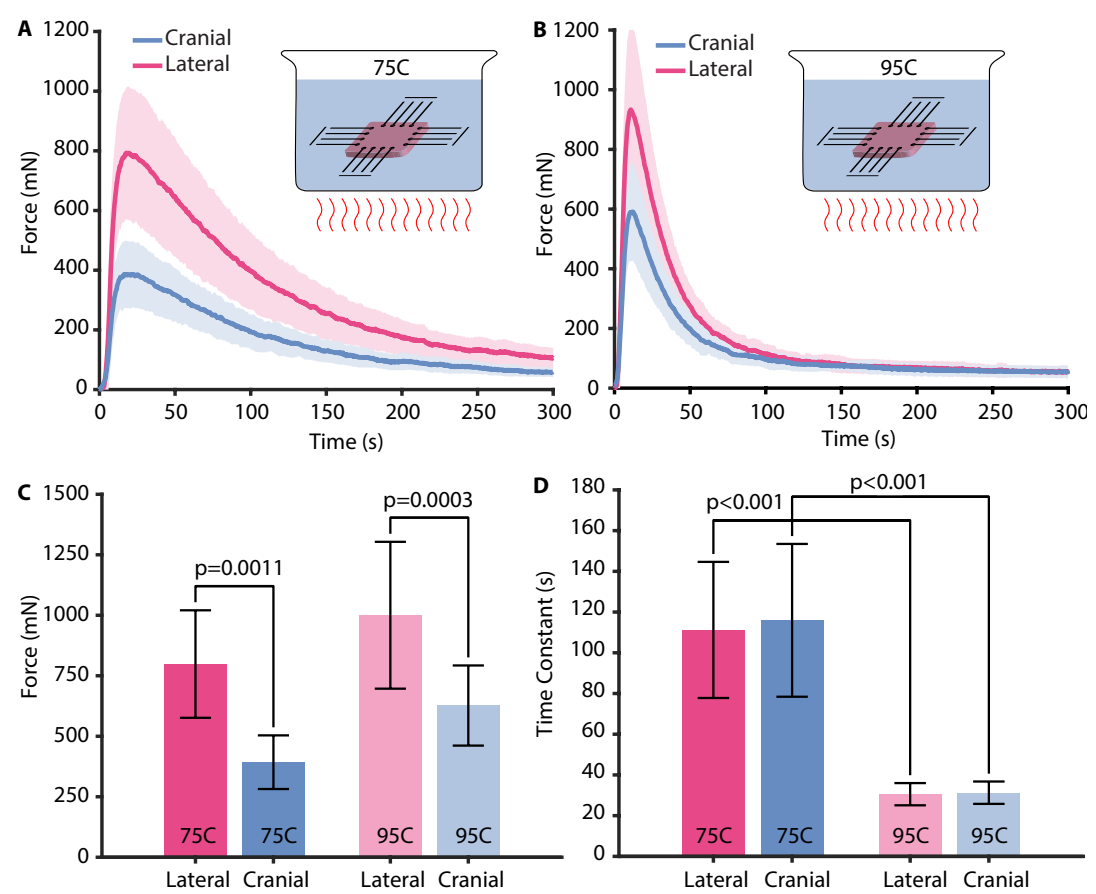

Figure 4: Heat treatment with $75^{\circ} \mathrm{C}$ and $95^{\circ} \mathrm{C} 1 \times \mathrm{xBS}$ induces anisotropic forces under isometric conditions. A) In both lateral and cranial direction, heat treatment with $75^{\circ} \mathrm{C}(\mathrm{n}=9)$ 1xPBS induced forces that rose quickly before exponentially decaying to almost zero within 5 minutes. Forces were higher in lateral than cranial direction (see C). B) Heat treatment with $95^{\circ} \mathrm{C}(\mathrm{n}=9) 1 \mathrm{xPBS}$ qualitatively mimicked the response to $75^{\circ} \mathrm{C}$. C) Heat treatment with $95^{\circ} \mathrm{C}$ reached higher peak forces than with $75^{\circ} \mathrm{C}$ (note, for clarity we omitted the lines indicating this difference: $\mathrm{p}=0.0064$ and $\mathrm{p}=0.0031$ in lateral and cranial directions, respectively) and forces were higher in lateral direction than cranial direction for both $75^{\circ} \mathrm{C}$ and $95^{\circ} \mathrm{C}$. D) Additionally, peak forces in response to $95^{\circ} \mathrm{C}$ heat treatment decayed faster

\section{Results}

We tested a total of 141 skin samples in our isotonic and isometric biaxial tests and our imaging protocols. The results of our isotonic tests are summarized in Figure 3. Overall, we found that skin shrinks when treated with 1xPBS of $75^{\circ} \mathrm{C}$ and $95^{\circ} \mathrm{C}$, but not when treated with $37^{\circ} \mathrm{C}$ and $55^{\circ} \mathrm{C} 1 \mathrm{xPBS}$, see Figures 3A-C. When samples shrunk, they shrunk more in lateral direction than in cranial direction $\left(\mathrm{p}<0.001\right.$ for both $75^{\circ} \mathrm{C}$ and $\left.95^{\circ} \mathrm{C}\right)$, see $3 \mathrm{D}$. This shrinkage appeared to occur at a faster rate at $95^{\circ} \mathrm{C}$ than at $75^{\circ} \mathrm{C}$, but in both cases equilibrated to similar strains $(\mathrm{p}=0.4844$ and $\mathrm{p}=0.3870$ in cranial and lateral direction, respectively). Thus, upon heat treatment skin shrinks anisotropically and at a rate that is temperature-dependent.

In addition to isotonic biaxial tests, we also conducted isometric biaxial tests.

${ }_{150}$ Those tests are summarized in Figure 4. Here we found that when constrained at its boundaries, skin produced significant forces upon exposure to $75^{\circ} \mathrm{C}$ and 

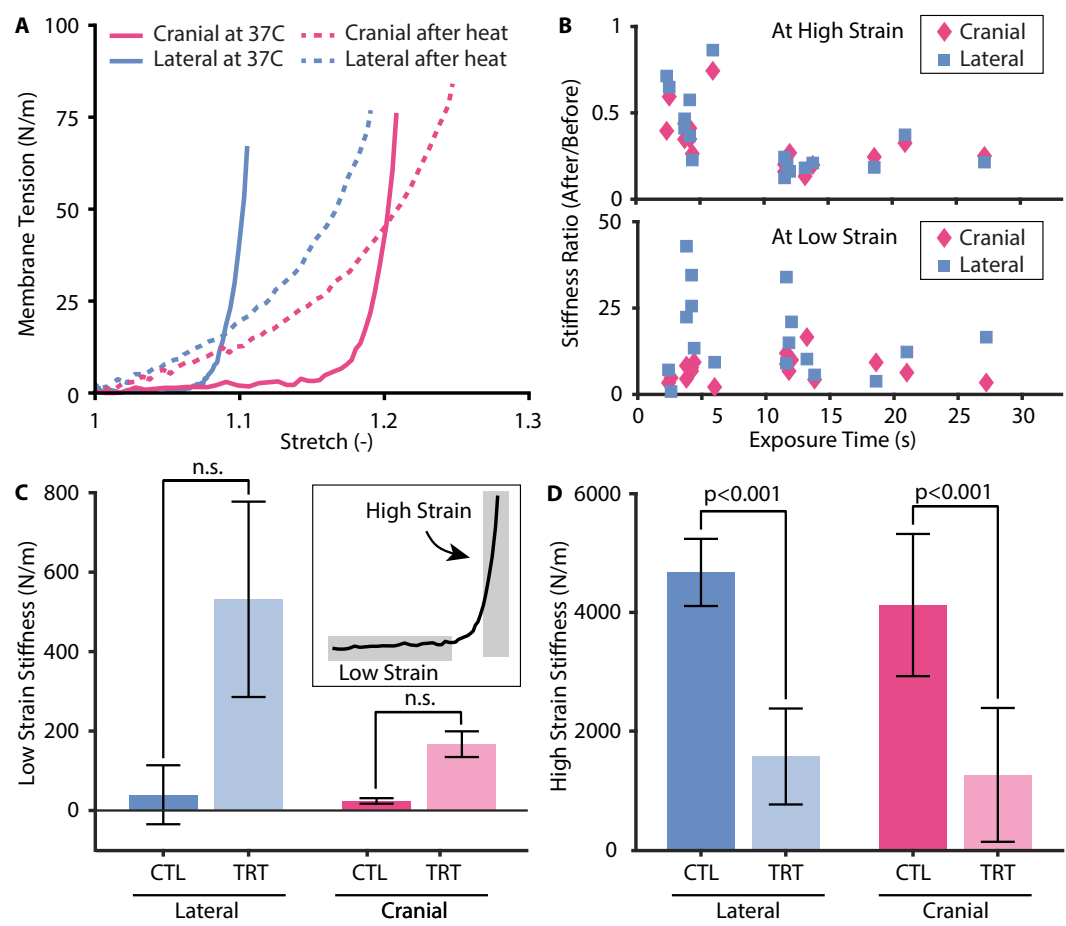

Figure 5: Heat treatment with $90^{\circ} \mathrm{C} 1 \mathrm{xPBS}$ alters constitutive behavior of skin. A) Example raw data of biaxial test before and after heat treatment for 10 seconds. B) The relationship of heat treatment duration and stiffness increase (at low strain) or decrease (at high strain) was subject to large variations and appeared uncorrelated $(n=17)$. C) When grouped, heat treatment-induced stiffening of skin at low strain, and D) heat treatment-induced reduction in stiffness of skin at high strain

$95^{\circ} \mathrm{C} 1 \mathrm{xPBS}$ that decayed after an initial peak. In contrast, skin did not produce significant forces when exposed to $55^{\circ} \mathrm{C} 1 \mathrm{xPBS}$, Figures $4 \mathrm{~A}-\mathrm{B}$ and Supplementary Figure S1, respectively. Skin's response to $75^{\circ} \mathrm{C}$ and $95^{\circ} \mathrm{C} 1 \mathrm{xPBS}$ was also anisotropic in that forces where higher in lateral direction than in cranial direction $\left(\mathrm{p}=0.0011\right.$ and $\mathrm{p}=0.0003$ at $75^{\circ} \mathrm{C}$ and $95^{\circ} \mathrm{C}$, respectively). Additionally, the peak force also increased with temperature $(\mathrm{p}=0.0064$ and $\mathrm{p}=0.0031$ in lateral and cranial directions, respectively), see $4 \mathrm{C}$. The peak force was also reached significantly faster at $95^{\circ} \mathrm{C}$ than at $75^{\circ} \mathrm{C}(\mathrm{p}<0.001$ for both directions), but did not differ between directions $\left(\mathrm{p}=0.6696\right.$ and $\mathrm{p}=0.9158$ for $75^{\circ} \mathrm{C}$ and $95^{\circ} \mathrm{C}$, respectively), see Supplementary Figure S2. Additionally, the time constant with which the force decayed after reaching its peak was significantly shorter during the $95^{\circ} \mathrm{C}$ treatment than after the $75^{\circ} \mathrm{C}$ treatment $(\mathrm{p}<0.001$ for both temperatures), see Figure 4 $\mathrm{D}$. The decay time, like the time to peak, did not differ with direction $\left(\mathrm{p}=0.6934\right.$ and $\mathrm{p}=0.9379$ for $75^{\circ} \mathrm{C}$ and $95^{\circ} \mathrm{C}$, respectively). Thus, upon heat treatment constrained skin produces significant anisotropic forces at rise and decay times that are temperature-dependent. Note, after five minutes 
of exposure to $75^{\circ} \mathrm{C}$ and $95^{\circ} \mathrm{C}$, these samples could not be tested mechanically. They had softened to the point where stretching them resulted in tear-outs from our fixtures. In contrast, five minutes of exposure to $55^{\circ} \mathrm{C}$ rendered the samples testable and only marginally affected skin's mechanics (see Supplementary Figure S3).

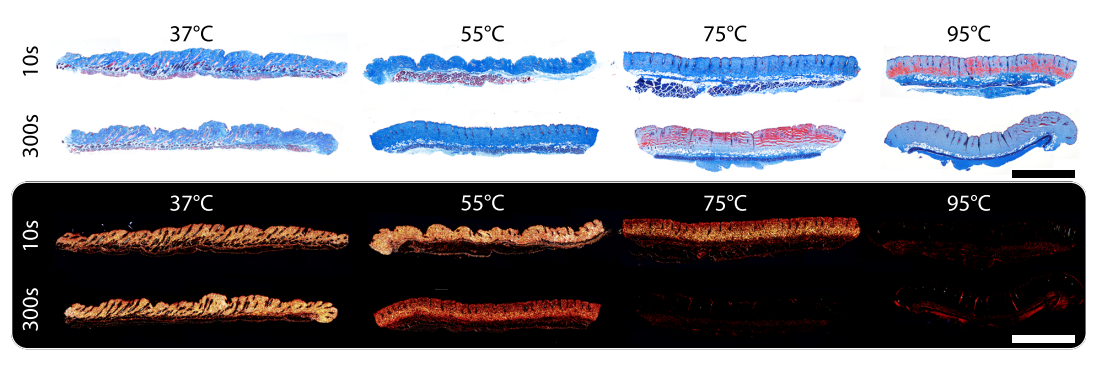

Figure 6: Heat treatment with $75^{\circ} \mathrm{C}$ and $95^{\circ} \mathrm{C} 1 \mathrm{xPBS}$ denatures collagen as demonstrated by loss of birefringence. This effect is time- and temperature-dependent as demonstrated by residual birefringence after $10 \mathrm{~s}$ of treatment with $75^{\circ} \mathrm{C}$, but loss of birefringence after 5 minutes, while heat treatment with $95^{\circ} \mathrm{C}$ resulted in loss of birefringence at $10 \mathrm{~s}$ and 5 minutes. We confirmed each time-temperature-pair with an independent set of samples (not shown). Scale bar $=1 \mathrm{~mm}$

Thus, in another set of experiments we exposed skin to $90^{\circ} \mathrm{C} 1 \mathrm{xPBS}$, but interrupted the force decay by quenching (i.e., quickly cooling with $37^{\circ} \mathrm{C} 1 \mathrm{xPBS}$ ) samples after exposure times between 0 and 30 seconds. Upon cooling, forces remained approximately constant rather than continuing to decay indicating that the internal damage processes had been interrupted, see Supplementary Video 1. After quenching, we tested those samples biaxially - in addition to the biaxial tests we conducted before the heat treatment. A representative biaxial 180 data set before and after 10 seconds of heat treatment (on the same sample) anecdotally demonstrates that heat treatment stiffened skin at low strains, but reduced its stiffness at high strain, see Figure $5 \mathrm{~A}$. Interestingly, we found that stiffening and softening at low and high strains, respectively, was not clearly correlated to the exposure times in the interval between 0 and 30 seconds, see 185 Figure $5 \mathrm{~B}$. When grouping the data - in other words, ignoring exposure time - we could demonstrate a statistically significant skin softening after heat treatment at high strains ( $\mathrm{p}<0.001$ in both lateral and cranial direction), but failed to show statistically significant stiffening after heat treatment at low strains $(\mathrm{p}=0.061$ and $\mathrm{p}=0.5348$ in lateral and cranial direction, respectively), see Figures 5 C-D. Thus, skin does soften at high strains and may stiffen at low strains when treated with $90^{\circ} \mathrm{C} 1 \mathrm{xPBS}$, where the latter phenomenon remains to be demonstrated.

To identify the source of the heat-induced changes to skin illustrated in Figures 355. we investigated (micro-)structural changes in our skin samples using linear and nonlinear optical methods. Specifically, we stained samples after iso-

195 tonic heat exposure to $37^{\circ} \mathrm{C}, 55^{\circ} \mathrm{C}, 75^{\circ} \mathrm{C}$, and $95^{\circ} \mathrm{C}$ with picrosirius red (and for reference with Masson trichrome). We found that the (birefringent) picrosirius red signal remained strong after exposure to $37^{\circ} \mathrm{C}$ and $55^{\circ} \mathrm{C}$, but vanished 

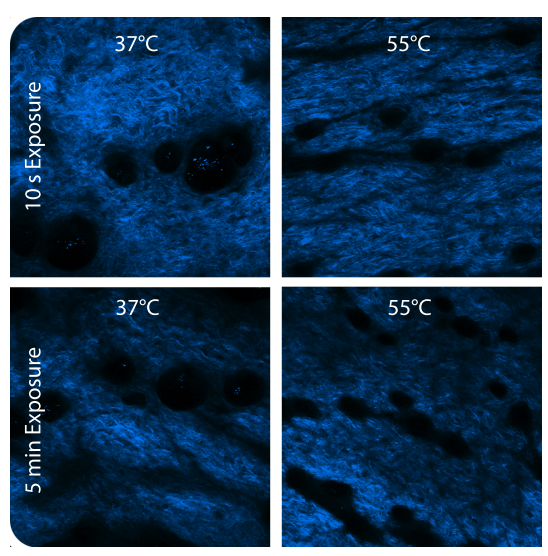

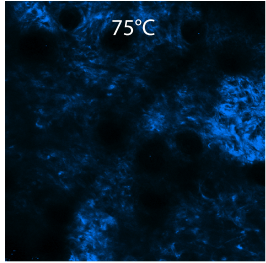

$75^{\circ} \mathrm{C}$

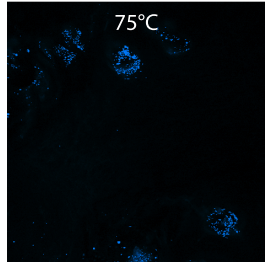

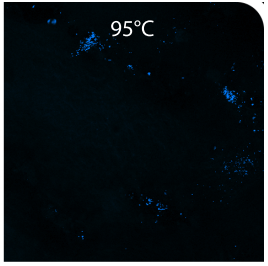

$95^{\circ} \mathrm{C}$

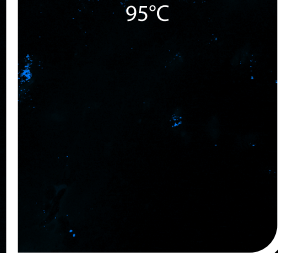

Figure 7: Heat treatment with $75^{\circ} \mathrm{C}$ and $95^{\circ} \mathrm{C} 1 \mathrm{xPBS}$ denatures collagen as demonstrated by loss of second harmonic generation (SHG) signal. This effect is time- and temperaturedependent as demonstrated by residual SHG signal after 10 seconds of treatment with $75^{\circ} \mathrm{C}$, but loss of SHG signal after 5 minutes, while heat treatment with $95^{\circ} \mathrm{C}$ resulted in loss of SHG signal at 10 seconds and 5 minutes. Note, we confirmed each time-temperature-pair with an independent set of samples (not shown). Image size $500 \mu \mathrm{m} \times 500 \mu \mathrm{m}$

after 5 minutes of $75^{\circ} \mathrm{C}$ exposure and 10 seconds as well as as 5 minutes of $95^{\circ} \mathrm{C}$ exposure, see Figure 6. Thus, heat treatment of skin removes the source of birefringence in a time- and temperature-dependent manner. Similarly, we imaged skin samples after the same isotonic heat treatment in our two-photon microscope and collected its SHG signal. We found that the SHG signal remains strong after exposure to $37^{\circ} \mathrm{C}$ and $55^{\circ} \mathrm{C}$, but vanished after 5 minutes of $75^{\circ} \mathrm{C}$ exposure and 10 seconds as well as 5 minutes of $95^{\circ} \mathrm{C}$ exposure, see Figure 7 . Thus, heat treatment of skin also removes the source of SHG in a time- and temperature-dependent manner and supports our histological findings.

\section{Discussion}

In our current work, we investigated the biaxial mechanics of skin after treatment with $37^{\circ} \mathrm{C}, 55^{\circ} \mathrm{C}, 75^{\circ} \mathrm{C}$, and $95^{\circ} \mathrm{C}$. Our work was motivated by missing information on how skin's anisotropic microstructure and mechanical constitutive behavior impacts its thermo-mechanical response to heat. We designed our experiments to investigate the thermo-mechanical response of skin to both isotonic loading - that is, traction-free - and isometric loading - that is, with fixed boundaries - in a temperature- and time-dependent manner. Our find-

215 ings confirmed many previous studies on the native, i.e., not heated, anisotropic microstructure and mechanical constitutive behavior of skin [29]. Additionally, our results were consistent with non-directionally-dependent previous studies on skin's thermo-mechanical response to heat treatment [22, 23. In addition to confirming previous findings, we unveiled significant, anisotropic thermomechanical effects in skin's response to heat treatment. We also used linear and 
nonlinear microscopy to confirm that the thermo-mechanical response of our samples was not temporary but followed from molecular damage.

\section{Summary of findings}

Our main findings were that heat treatment leads to significant skin shrink-

225 conditions. Under isometric conditions, skin also softens at large strains. These effects are temperature-, time-, and direction-dependent. Specifically, our findings on temperature-dependence are: i) Under isotonic conditions, heat treatment induces skin shrinkage at temperatures larger than $55^{\circ} \mathrm{C}$; ii) Under isotonic 230 conditions, higher temperatures induce faster shrinkage, but do not lead to more shrinkage; iii) Under isometric conditions, heat treatment induces forces at temperatures larger than $55^{\circ} \mathrm{C}$; iv) Under isometric conditions, higher temperature induces forces faster, lead to larger forces that also decay faster. Our findings on time-dependence are: i) Under isotonic conditions, shrinkage sets in within 235 seconds but then equilibriates; ii) Under isometric conditions, force production peaks within seconds, but then continuously decays until dissipated. Finally, our findings on direction-dependence are: i) Under isotonic conditions, heat treatment $\left(>55^{\circ} \mathrm{C}\right)$ induces more shrinkage in lateral direction than in cranial direction; ii) Under isometric conditions, heat treatment $\left(>55^{\circ} \mathrm{C}\right)$ induces conditions, heat treatment $\left(>55^{\circ} \mathrm{C}\right)$ reduces mechanical anisotropy.

Based on our histological and two-photon based analysis, skin's thermomechanical response to heat treatment was correlated with a degradation of optical properties - birefringence and SHG - that are related to collagen's struc245 ture [30, 31]. In other words, it is, at least in part, the heat treatment-induced denaturing of collagen that leads to above thermo-mechanical phenomena.

\section{Hypothetical model of skin's thermo-mechanical response to heat treatment}

Combining our thermo-mechanical and optical observation, we suggest the following hypothetical model for the effects of heat treatment on skin: Thermal 250 destruction of intra- and inter-molecular bonds in the collagen molecule lead to the disruption of collagen's rod-like alpha helix 32. Without these bonds, coiling results in an energetically favorable state and thus leads to tissue shrinkage in the absence of external forces 33 . Phenomenologically, this transition may be described as redefinition of the stress-free reference state of the colla255 gen molecule to shorter lengths 34 . The increased density of collagen in the lateral direction in comparison to the cranial direction amplifies this effect in a directionally-dependent manner [35. The thermal destruction of the intraand inter molecular bonds is likely driven by an Arrhenius-type, time-dependent mechanism, thus explaining the increased rate of shrinkage at higher tempera260 tures [5]. In the presence of constraints, the same thermal destruction of intraand inter-molecular bonds occurs. However, external tractions prevent coiling of the collagen molecular, thus, leading to the production of forces as the stress-free reference configuration of the collagen molecule shifts to shorter lengths. Here, 
again, anisotropic distributions of collagen result in a direction-dependent number of coiling collagen fibers and thus a higher production of force in the lateral direction than the cranial direction. Also, here, again, Arrhenius-type reactions lead to a temperature dependent production of these forces. More mysterious, however, is the specific temporal evolution of the force production. If the forces were produced through the constrained coiling of collagen, why do they decay 270 over time? We propose two alternative models. First, we propose that continued exposure to heat may additionally degrade collagen. In turn, collagen may entered a third, further degraded state (beyond being coiled). Second, and alternatively, it may be that, under force, the denatured collagen molecule may undergo viscoelastic stress-relaxation. In either case, time-dependence of the 275 additional denaturation or, alternatively, viscoelastic stress-relaxation, leads to faster decay times at higher temperatures. This hypothetical model also explains our observation that heat treated skin anecdotally stiffens at low strains and softens at high strains. Under native, pre-treated conditions, wavy collagen fibers are activated only at large strains, a mechanism that lends skin - and 280 many other collageneous soft tissues - their classic J-shaped or strain stiffening stress-strain behavior [36. Upon heat treatment, the reduced reference configuration of collagen leads in our hypothetical model to an early activation of its contribution to the tissue's resistance to external loading and thus a stiffening at small strains. On the other hand, the destruction of inter- and intra-molecular 285 bonds reduces collagen's load-bearing capacity and leads to a softening at large strains.

Shortcomings, unexplained phenomena, and future work

We believe that our work is an important step toward understanding the biaxial mechanics of thermally denaturing skin. However, our work is naturally 290 subject to limitations and there is a great need for additional work. For example, Chen et al have shown that the thermo-mechanics of collageneous tissues are not only time-, and temperature-dependent, but also load-dependent 7 . This additional determinant of skin's response to thermal treatment should, in the future, be added to our observed dependence on time, temperature, and di295 rection. From an experimental design perspective, we observed the mechanical manifestations to thermal treatment - shrinkage, force production, and change in constitutive behavior - and the microstructural origins - denaturation of collagen - independently. Future studies should aim at a design that simultaneously images skin's microstructure while recording its mechanical response to heat treatment [37. Additionally, we failed to demonstrate a statistical relationship between tissue stiffening and softening - at low and high strains, respectively - and heat exposure times. Our failure to do so is most likely related to the high-rate, nonlinear temperature response curve as seen in the time evolution of force production. Future experiments toward establishing a clear relationship 305 between skin stiffening/softening and exposure times will have to take great care to control exposure times more accurately than we were able to do in this present study. Finally, and most importantly, we have posed a theoretical model connecting skin's thermo-mechanical response to heat treatment to its 
microstructural origins. As a first step toward confirming this model, we will implement this model numerically and test its ability to predict our observations.

\section{Conclusion}

We conclude that thermal treatment of skin above $55^{\circ} \mathrm{C}$ results in time, temperature-, and direction-dependent shrinkage under isotonic conditions. 315 The largest shrinkage occurs in the direction of the predominant collagen orientation. Similarly, thermal treatment of skin above $55^{\circ} \mathrm{C}$ results in time-, temperature-, and direction-dependent force production under isometric conditions. The largest forces occur in the direction of the predominant collagen orientation. Additionally, we conclude that thermal treatment of skin results in softening of skin at high strains. These thermo-mechanical phenomena are driven by the denaturation of collagen as demonstrated through histology and two-photon microscopy. This work is a critical step toward developing a fundamental understanding of the relationship between skin microstructure, mechanics, and its response to thermal treatment. Ultimately, this knowledge will

325 be critical to optimally treating burn wounds, designing medical devices, and optimizing aesthetic modifications.

\section{Acknowledgements}

We acknowledge the National Science Foundation for their partial support of this project via Grant \#1916663 (Rausch, Buganza), the National Institutes of Health for their partial support of this project via Grant F31HL145976 (Meador), and the University of Texas at Austin Graduate School Continuing Student Fellowship (Sugerman).

\section{Disclosures}

Dr. Rausch has a speaking agreement with Edwards Lifesciences. None of the other authors have any potential conflicts of interest.

\section{References}

[1] C. A. Miles, N. C. Avery, V. V. Rodin, A. J. Bailey, The increase in denaturation temperature following cross-linking of collagen is caused by dehydration of the fibres, Journal of molecular biology 346 (2) (2005) 551-556.

340 [2] C. Miles, A. Bailey, Thermally labile domains in the collagen molecule, Micron 32 (3) (2001) 325-332.

[3] L. Marshall, A. Tarakanova, P. Szarek, D. M. Pierce, Cartilage and collagen mechanics under large-strain shear within in vivo and at supraphysiogical temperatures, Journal of the mechanical behavior of biomedical materials 103 (2020) 103595. 
[4] N. Wright, J. Humphrey, Denaturation of collagen via heating: an irreversible rate process, Annual review of biomedical engineering 4 (1) (2002) $109-128$.

[5] S. S. Chen, N. T. Wright, J. D. Humphrey, Phenomenological evolution

[15] L. J. Davidson, J. Luna, R. Virmani, J. A. Asscher, C. J. Davidson, First human experience of thermal arterial closure, Catheterization and Cardiovascular Interventions 84 (1) (2014) 30-36. 
[16] J. Humphrey, Continuum thermomechanics and the clinical treatment of disease and injury, Appl. Mech. Rev. 56 (2) (2003) 231-260.

[17] G. Limbert, Skin Biophysics: From Experimental Characterisation to Advanced Modelling, Vol. 22, Springer, 2019.

[18] L. A. Brightman, J. A. Brauer, R. Anolik, E. Weiss, J. Karen, A. Chapas, E. Hale, L. Bernstein, R. G. Geronemus, Ablative and fractional ablative lasers, Dermatologic clinics 27 (4) (2009) 479-489.

[19] R. Karamanoukian, C. Ukatu, E. Lee, J. Hyman, M. Sundine, M. Kobayashi, G. R. Evans, Aesthetic skin branding: a novel form of body art with adverse clinical sequela, Journal of burn care \& research 27 (1) (2006) 108-110.

[20] R. C. Webb, R. M. Pielak, P. Bastien, J. Ayers, J. Niittynen, J. Kurniawan, M. Manco, A. Lin, N. H. Cho, V. Malyrchuk, et al., Thermal transport characteristics of human skin measured in vivo using ultrathin conformal arrays of thermal sensors and actuators, PloS one 10 (2) (2015) e0118131.

[21] A. McBride, S. Bargmann, D. Pond, G. Limbert, Thermoelastic modelling of the skin at finite deformations, Journal of thermal biology 62 (2016) 201-209.

[22] B. Zhou, F. Xu, C. Chen, T. Lu, Strain rate sensitivity of skin tissue under thermomechanical loading, Philosophical Transactions of the Royal Society A: Mathematical, Physical and Engineering Sciences 368 (1912) (2010) 679-690.

[23] F. Xu, T. Lu, K. Seffen, Thermally-induced change in the relaxation behavior of skin tissue, Journal of biomechanical engineering 131 (7).

[24] B. Lynch, S. Bancelin, C. Bonod-Bidaud, J.-B. Gueusquin, F. Ruggiero, M.-C. Schanne-Klein, J.-M. Allain, A novel microstructural interpretation for the biomechanics of mouse skin derived from multiscale characterization, Acta biomaterialia 50 (2017) 302-311.

[25] W. D. Meador, M. Mathur, G. P. Sugerman, T. Jazwiec, M. Malinowski, M. R. Bersi, T. A. Timek, M. K. Rausch, A detailed mechanical and microstructural analysis of ovine tricuspid valve leaflets, Acta biomaterialia 102 (2020) 100-113.

[26] W. D. Meador, M. Mathur, G. P. Sugerman, M. Malinowski, T. Jazwiec, X. Wang, C. M. Lacerda, T. A. Timek, M. K. Rausch, The tricuspid valve also maladapts as shown in sheep with biventricular heart failure, Elife 9 (2020) e63855.

[27] W. D. Meador, G. P. Sugerman, H. M. Story, A. W. Seifert, M. R. Bersi, A. B. Tepole, M. K. Rausch, The regional-dependent biaxial behavior of young and aged mouse skin: A detailed histomechanical characterization, 
residual strain analysis, and constitutive model, Acta biomaterialia 101 (2020) 403-413.

425

[28] H. Singmann, B. Bolker, J. Westfall, F. Aust, M. S. Ben-Shachar, afex: Analysis of factorial experiments, R package version 0.13-145.

[29] A. Pissarenko, C. J. Ruestes, M. A. Meyers, Constitutive description of skin dermis: Through analytical continuum and coarse-grained approaches for multi-scale understanding, Acta biomaterialia 106 (2020) 208-224.

430

[30] S. Thomsen, J. A. Pearce, W.-F. Cheong, Changes in birefringence as markers of thermal damage in tissues, IEEE Transactions on Biomedical engineering 36 (12) (1989) 1174-1179.

[31] Y. Sun, W.-L. Chen, S.-J. Lin, S.-H. Jee, Y.-F. Chen, L.-C. Lin, P. T. So, C.-Y. Dong, Investigating mechanisms of collagen thermal denaturation by high resolution second-harmonic generation imaging, Biophysical journal 91 (7) (2006) 2620-2625.

[32] B. Brodsky, A. V. Persikov, Molecular structure of the collagen triple helix, Advances in protein chemistry 70 (2005) 301-339.

[33] E. Andronikashvili, G. Mrevlishvili, G. Sh. Japaridze, V. Sokhadze, K. Kvavadze, Thermal properties of collagen in helical and random coiled states in the temperature range from 4 to $300 \mathrm{k}$, Biopolymers: Original Research on Biomolecules 15 (10) (1976) 1991-2004.

[34] M. K. Rausch, J. D. Humphrey, A microstructurally inspired damage model for early venous thrombus, Journal of the mechanical behavior of biomed-

[35] S. Bancelin, B. Lynch, C. Bonod-Bidaud, G. Ducourthial, S. Psilodimitrakopoulos, P. Dokládal, J.-M. Allain, M.-C. Schanne-Klein, F. Ruggiero, Ex vivo multiscale quantitation of skin biomechanics in wild-type and genetically-modified mice using multiphoton microscopy, Scientific reports 5 (1) (2015) 1-14.

[36] S. Chen, A. N. Annaidh, S. Roccabianca, A microstructurally inspired constitutive model for skin mechanics, Biomechanics and modeling in mechanobiology 19 (1) (2020) 275-289.

[37] P. Wells, S. Thomsen, M. Jones, S. Baek, J. Humphrey, Histological evidence for the role of mechanical stress in modulating thermal denaturation of collagen, Biomechanics and modeling in mechanobiology 4 (4) (2005) 201-210. 